unterschiedliche Skalierung der Jahreszahlen. Hier wäre ein sorgfältigeres Lektorat wünschenswert gewesen.

Insgesamt legen Grell und Lammert eine umfassende und strukturierte Einführung in das fragmentierte Politikfeld der US-amerikanischen Sozialpolitik vor. Dabei beweisen die Autoren ihre tiefen Kenntnisse der Materie sowie der aktuellen wissenschaftlichen und gesellschaftlichen Diskussionen um den amerikanischen Wohlfahrtsstaat. Insbesondere die von den Autoren gewählte weite Definition von wohlfahrtsstaatlichen Leistungen überzeugt, eröffnet sie doch Einblicke in versteckte Bereiche amerikanischer Sozialpolitik und hinterfragt die These des schwachen Staates in den USA. So ist das Buch für die universitäre Lehre, aber auch für ein größeres, politischinteressiertes Publikum, zu empfehlen.

Markus B. Siewert

\section{INTERNATIONALE BEZIEHUNGEN}

\section{Sammelrezension}

Bellamy, Alex J. und Paul D. Williams. Understanding Peacekeeping. 2. Aufl. Cambridge. Polity Press 2010. 447 Seiten. 25,30€.

Benner, Thorsten, Stephan Mergenthaler und Philipp Rotmann. The New World of UN Peace Operations: Learning to Build Peace? Oxford. Oxford University Press 2011. 247 Seiten. 75,70 €.

\author{
Bellamy, Alex J. und Paul D. Williams \\ (Hrsg.). Providing Peacekeepers: The \\ Politics, Challenges, and Future of \\ United Nations Peacekeeping Contribu- \\ tions. Oxford. Oxford University Press \\ 2013. 459 Seiten. $96,30 €$.
}

Theoretisch zu denken meint das Bemühen, jedes Einzelereignis einer Klasse von Ereignissen zuzuordnen (James Rosenau). So gesehen kann ein erhebliches Theoriedefizit bei der wissenschaftlichen Analyse von UN-Friedensmissionen konstatiert werden. Das beginnt mit dem begrifflichen Instrumentarium zur Beschreibung solcher Missionen. Friedensprävention, -erhaltung, -überwachung, -erzwingung, oder -stabilisierung usw. - eine konsistente und trennscharfe Verwendung dieser Begriffe hat sich bis heute weder in der Praxis, noch in der Wissenschaft eingestellt. Damit zusammenhängend wird eine intensive Debatte um "richtige“ Friedensmissionen geführt.

Das inzwischen in der zweiten Auflage vorliegende Standardwerk Understanding Peacekeeping hilft diesbezüglich, die Übersicht zu erhalten. Hier wird gleich mehrfach eine Schneise durch den Begriffs- und Debattendschungel geschlagen. So zum Beispiel mit der Unterscheidung westfälischer versus postwestfälischer Programmatiken (36) oder im Zuge der Differenzierung lokaler, nationaler, regionaler, globaler und makrostruktureller Analyseebenen (21). Zudem referieren die Autoren Alex J. Bellamy und Paul D. Williams die historische Entwicklung der UN-Friedenspolitik (69-152) und fassen die wichtigsten gegenwärtigen Herausforderungen pointiert zusammen (Regionalisierung, Privatisierung, Schutz von Zivilisten, Berücksichtigung von Genderaspekten, Polizeimissionen). Zwar wird die anhand zahlreicher empirischer Fälle exemplifizierte Typologie aus sechs Missionstypen (Preventive Deployments, Traditional Peacekeeping, Wider Peacekeeping, Peace Enforcement, Assisting Transitions, Transitional Administrations, Peace Support 
Operations) wohl nicht überall Anklang finden. Doch unabhängig davon stellt Understanding Peacekeeping auch in der Neuauflage ein Grundlagenwerk im besten Sinne dar.

Ein Theoriedefizit erschwert auch die Auseinandersetzung mit den Ursachen des Zustandekommens von UN-Friedensmissionen, ihrer Merkmale sowie ihrer Ergebnisse. Selten werden dabei Gemeinsamkeiten und Regelmäßigkeiten über den Einzelfall hinaus in den Blick genommen. Zwei Ausnahmen sind hervorzuheben.

Thorsten Benner, Stephan Mergenthaler und Philipp Rotmann untersuchen in insgesamt zwölf Fallstudien aus vier Bereichen (Polizeireform, Justizreform, Reintegration und interadministrative Zusammenarbeit) die Voraussetzungen, Erfolge und Grenzen organisatorischen Lernens im Rahmen der UN-Bürokratie. Um eines vorwegzunehmen: Es handelt sich um ein eindrucksvolles Projekt, das eine wichtige Forschungslücke schließt. Moderne Friedensmissionen, so die Ausgangsüberlegung der Autoren, sind überaus komplex - bedingt durch ambitionierte Ziele (Wiederaufbau, Rechtstaatlichkeit, Menschenrechtsschutz) und ihr operatives Umfeld (in der Regel innerstaatliche oder transnationale Kriege) (14-25). Ihr Erfolg ist daher nicht zuletzt abhängig vom Einsatz adäquaten ökonomischen, politischen und kulturellen Wissens. Die benötigten Kompetenzen sind aber entweder gar nicht vorhanden, oder innerhalb der UN-Bürokratie verstreut (22). Das erschwert ein effektives Wissensmanagement. Dazu kommen chronische Finanzknappheit und Aufgabenüberlastung, eine hohe Personalfluktuation und eine gegen Standardisierung eingestellte Organisationskultur. Erst sehr spät, nämlich in den 2000er Jahren, wurden im Department for Peacekeeping Operations (DPKO) allmählich Mechanismen für den horizontalen und vertikalen Austausch missionsübergreifend relevanter Erfahrungen etabliert und Best-Practices systematisch in Standards und Doktrinen festgehalten (39-50). Mit anderen Worten: Es wurde in das investiert, was die Autoren eine „Infrastruktur des Lernens" (50) nennen. Im selben Jahrzehnt hat die UN-Bürokratie eine Reihe substantieller „Lektionen“ tatsächlich akzeptiert, institutionalisiert und implementiert: Dazu zählen etwa kurzfristig abrufbare Polizeikapazitäten (83-92) oder der Aufbau interadministrativer Führungsstrukturen im Einsatz (180-187). In anderen Fällen blieb der Lernprozess allerdings bereits auf der Stufe der Kritik und Debatte stecken. So etwa in der Frage des Umgangs mit traditionellen Rechtspraktiken in Post-Konflikt-Gesellschaften (135142). Wieder andere Lektionen wurden schließlich zwar organisationsweit akzeptiert und kodifiziert, es haperte aber bei der Umsetzung. An dieser letzten Hürde scheiterte etwa die Berücksichtigung von Genderaspekten im Zuge von Reintegrationsmaßnahmen (163170). Wie ist nun diese beträchtliche Varianz zu erklären? Was sind die Ursachen des Scheiterns oder Gelingens eines Lernzyklus? Die Autoren unterscheiden zwei Cluster von Faktoren: Infrastruktur (Kompetenzen, Ressourcen, Routinen, Anreize, Organisationskultur) und Politics (Führung, Interventionen von Mitgliedsstaaten, bürokratischer Wettbewerb) (59-64). Im Ergebnis zeigt sich: Politische Faktoren haben das größte Gewicht. So werden Lernprozesse oft durch bürokratische Grabenkämpfe oder Kontroversen zwischen den Mitgliedsstaaten blockiert. 
Andererseits kann die Unterstützung des Lernzyklus durch hochrangige UNVertreter oder eine Koalition von diversen Akteuren den Reformprozess zum Erfolg führen. Kommt es indes nicht zur Politisierung des Prozesses, sind die Spezifika der vorhandenen Lerninfrastruktur oftmals ausschlaggebend. Sie kann die Diffusion neuer Erkenntnisse unterstützen oder aber behindern. Daraus folgern die Autoren eine wichtige praktische Konsequenz: Die bisherigen institutionellen Reformen sind zwar kein Allheilmittel, aber sie zeigen doch oftmals positive Effekte. Entsprechend sollten derartige Bemühungen fortgesetzt werden.

Abgesehen vom analytischen und praktischen Nutzen des Bandes vermitteln die prägnanten Beschreibungen der Einsatzrealitäten einen guten Eindruck von den immensen Herausforderungen gegenwärtiger Friedensmissionen. Ein gutes Beispiel ist die gesellschaftliche Reintegration ehemaliger Kämpfer (146-172). Wenn diesen keine Perspektiven eröffnet werden, stehen sie als Reservoir für erneute Anwerbungsversuche durch regionale Kriegsparteien zur Verfügung. Genau das geschah 2003 mit der Anwerbung beschäftigungsloser Kämpfer aus Sierra Leone durch Kriegsparteien in Liberia. Gleichsam dürfen Aufbauhilfemaßnahmen nicht die lokalen Gemeinschaften, in die die Kämpfer zurückkehren sollen, vernachlässigen. Denn sonst wird der Täterstatus von Seiten der Opfer mit Privilegien assoziiert, und das gesamte Reintagrationsprogramm trifft auf Ablehnung. Diese Lektion war weder offensichtlich, noch ist sie einfach in praktische Politik $\mathrm{zu}$ übersetzen. Umso mehr verdienen die Bemühungen der UN Respekt. Wie lässt sich der Band abschließend charakterisieren? Sicher ist es keine für
Proseminare geeignete Einführung in das Themengebiet. Doch ist er ebenso wenig eine nur Experten zugängliche und mit Akronymen vollgepackte Dokumentenanalyse. Zur Anschaulichkeit des Bandes tragen nicht zuletzt die 250, meist anonymen, Interviews mit UNFunktionären, Diplomaten und Einsatzverantwortlichen „im Feld“ (unter anderem in Liberia, Osttimor und auf Haiti) bei. So kann der Text auch dem interessierten Laien als Vertiefung in das Themengebiet dienen. Mit Blick auf den theoretischen Erkenntnisgewinn des Bandes irritiert allerdings die suggerierte Distanz zum Mainstream organisationstheoretischer Forschung. Statt Organisationen als prinzipiell lernunfähig anzusehen (57-58), würden viele Organisationstheoretiker wohl eher den inkrementellen Charakter des Organisationslernens hervorheben und die Ausrichtung an zufrieden stellenden (statt optimalen) Ergebnissen. Sind die Ergebnisse der Autoren dann nicht eher als eine Bestätigung denn als Abweichung von organisationstheoretischen Erwartungen zu sehen?

Nicht auf der Ebene der internationalen Politik bzw. internationaler Organisationen, sondern auf der Ebene der Außenpolitikanalyse ist der von Alex J. Bellamy und Paul D. Williams herausgegebene Sammelband Providing Peacekeepers anzusiedeln. Beleuchtet werden das zunehmende oder abnehmende Engagement wichtiger Truppen bereitstellender Staaten. Aus einer Art Vogelperspektive widmen sich die ersten beiden Kapitel zunächst übergreifenden Trends. Einige wenige, vor allem südasiatische Entwicklungs- und Schwellenländer (Bangladesch, Pakistan, Indien) stellen demnach das Gros der UN-Blauhelmkontingente. Westliche Industriestaaten hingegen leisten 
zwar mitunter politische und finanzielle Unterstützung. Ihre Soldaten unterstellen sie aber immer seltener einem UN-Kommando. Stattdessen präferieren sie UN-mandatierte, aber von der NATO oder der EU implementierte Missionen. Im Ergebnis mangelt es den genuinen UN-Friedensmissionen an Spezialkräften und hochwertigem Equipment. Die Zeichen für eine signifikante Verbreiterung der Truppenstellerbasis stehen indes nicht gut. Auch weil mehr und mehr UN-Mitgliedsstaaten das Instrument symbolischer Kleinstbeiträge (etwa die Entsendung einiger weniger Militärbeobachter) für sich entdeckt haben (47-67). Über solche Trends hinaus adressiert der Band die Ursachen für die beträchtliche Varianz des UN-Engagements selbst ökonomisch und militärisch gleichrangiger Staaten. Zunächst wird der Erklärungsanspruch etablierter Paradigmen der Internationalen Beziehungen und der Außenpolitikanalyse in dieser Frage zurückgewiesen. Das scheint nicht ganz berechtigt. Wegen einiger Anomalien (4-17) müssen sozialwissenschaftliche Theorien sicher nicht direkt über Bord geworfen werden. Bellamy und Williams geht es freilich um eine Art Neustart der Theoriebildung. Dafür geben sie den Autorinnen und Autoren der diversen Länderstudien des Bandes ein einfaches heuristisches Raster an die Hand. Für jeden nationalen Entscheidungskontext werden sicherheitspolitische, ökonomische, institutionelle und normative Beweggründe für (oder gegen) die Entsendung von Truppen in UN-Friedensmissionen plausibilisiert. Beleuchtet werden in 16 Fallstudien die Entsendepolitik der fünf Sicherheitsratsmitglieder, wichtiger Truppensteller (Bangladesch, Pakistan, Indien, Nigeria, Ghana, Nepal, Uruguay) so- wie einiger aktuell oder perspektivischer "Neulinge“ (Brasilien, Türkei, Südafrika, Japan). Alle Einzelfallstudien sind von ausgewiesenen Expertinnen und Experten verfasst worden. So wird erstmals hochrelevantes Wissen über den nationalen Entscheidungskontext von UN-Missionen an einem Ort zusammengetragen. Leider ist die Struktur der Einzelbeiträge nicht immer einheitlich. So erläutern beispielsweise viele Kapitel (Großbritannien, Russland, Bangladesch, Pakistan, Indien, Ghana, Brasilien, Türkei, Südafrika) detailliert relevante Entscheidungsprozesse, andere wiederum fokussieren stark auf die Erfahrungen einzelner Missionsbeteiligungen und sind stärker narrativ angelegt (Nigeria, Frankreich, Uruguay). Ebenfalls nicht ganz stringent fällt die Wahl empirischer Indikatoren für die Operationalisierung der Handlungslogiken aus. Das sind freilich typische Schwächen vieler Sammelbände und sollte nicht überbewertet werden. Zumal die Herausgeber in den Schlusskapiteln wieder eine stärkere Systematik anstreben. Inhaltlich heben sie den maßgeblichen Einfluss innerstaatlicher politischer Konstellationen und Wertvorstellungen, beispielsweise Traditionen eines außenpolitischen Exzeptionalismus oder den Einfluss und die Sichtweisen des Militärs, hervor (418). Als Destillat der Einzelfallstudien präsentieren sie dann einen Theorierahmen zur Synthese unterschiedlicher kausaler Faktoren (424434). Zentral ist die Unterscheidung zwischen langfristigen institutionellen und kulturellen Dispositionen und konkreten Politikmustern und -praktiken. Ganz trennscharf gelingt diese Unterscheidung freilich nicht. Zudem werden die bereits vorhandenen methodischen Angebote multikausaler 
Analysen gar nicht rezipiert. Zu nennen wären etwa Fuzzy Set Qualitative Comparative Analysis (QCA), kontrafaktische Analysen oder Forschungen im Rahmen der polyheuristischen Entscheidungstheorie.

Trotz solcher konzeptioneller Schwächen verspricht Providing Peacekeepers ein empirisches Standardwerk über nationale Entsendepolitiken zu werden. Mehr noch: Es könnte auch eine Initialzündung für eine stärker systematische und kumulative theoriegeleitete Forschung der UN-Politik Truppen stellender Staaten leisten. Diesbezüglich wäre allerdings eine stärkere Rezeption der Erkenntnisse, Methoden und Konzepte der Disziplin der Außenpolitikanalyse wünschenswert.

Mischa Hansel

\section{Skocpol, Theda. Obama and America's Political Future. Cambridge (USA)/ London (UK). Harvard University Press 2012. 195 Seiten. $27,95 €$.}

Der Titel dieses Bandes war gewiss nicht nur im US-Wahljahr 2012 von allgemeinem Interesse, sondern seine inhaltlichen Implikationen dürften inzwischen auch ihre Wirkung im Bereich der Amerikaforschung entfaltet haben. Das Buch der Politik- und Soziologieprofessorin Theda Skocpol von der Harvard University behandelt relevante innenpolitische Themen unter Barack Obama: die von ihr so bezeichnete „Halfway-New-Deal“-Politik des Präsidenten, die „New-Deal“-Fantasie einzelner US-Journalisten wie Peter Beinart, Obamas „Problem“ der Missdeutung seines politischen Mandats und die Herausforderung für den Präsidenten, seine Reformpolitik dem amerikanischen Volk zu vermitteln.
Neben Skocpol sind auch Larry M. Bartels (Vanderbilt University), Mickey Edwards (Aspen Institute; ehemaliges republikanisches Kongressmitglied) und Suzanne Mettler (Cornell University) an dem Werk beteiligt. Der Mix aus Beiträgen von Theoretikern und Praktikern macht diesen Band zu einer aufschlussreichen Lektüre der Entwicklungen in der US-Politik bis 2012, wie noch zu zeigen sein wird.

Das Buch gliedert sich in ein Vorwort von Daniel Carpenter (Harvard University), in zwei Beiträge von Skocpol - einen längeren zentralen Aufsatz und eine Replik auf die kritischen Kommentierungen ihrer Untersuchung sowie in drei Beiträge von Bartels, Edwards und Mettler, die es inhaltlich erweitern und abrunden. Kernstück des Bandes ist der Aufsatz „Obama's New Deal, Tea Party Reaction, and America's Political Future“ von Skocpol, der einem fein gesponnenen Argumentationsfaden folgt: Der erste Teil ihrer Analyse beleuchtet die politischen Ausgangsbedingungen und Begleiterscheinungen für Präsident Obamas Bemühungen um einen ,second New Deal“ (74) mit dem Ziel der Revision des Steuersystems und der Sozialprogramme in den USA, um bestehende Ungleichheiten $\mathrm{zu}$ beseitigen, gesellschaftliche Chancen zu erweitern und die soziale Sicherheit zu stärken. Den theoretischen Rahmen bildet in diesem Zusammenhang der sogenannte Historical Institutionalism mit seiner historisch-vergleichenden Methode, der den Staat als „Mitspieler“ in sozialen Konflikten begreift. Die Bedeutung der institutionellen Umgebung für politische Entscheidungen und Auseinandersetzungen über Politikinhalte ist dabei besonders zu berücksichtigen. Der zweite Teil der Analyse von Skocpol befasst 